\title{
5-Hydroxy-6-Methyluracil as a Polyvinyl Chloride Stabilizer
}

\author{
R.M. Akhmetkhanov ${ }^{1}$, I.T. Gabitov ${ }^{1}$, A.G. Mustafin ${ }^{1}$, V.P. Zakharov ${ }^{1}$ and G.E. Zaikov ${ }^{2, *}$ \\ ${ }^{1}$ Bashkir State University, 32 Zaki Validi St., 450076 Ufa, Russia \\ ${ }^{2}$ N.M. Emanuel Institute of Biochemical Physics, Russian Academy of Sciences, 4 Kosygin Str., 119334 \\ Moscow, Russia
}

\begin{abstract}
Kinetic regularities of thermooxidative dehydrochlorination of rigid and plasticized PVC in the presence of 5hydroxy-6-methyluracil have been studied. The high antioxidant efficacy of 5-hydroxy-6-methyluracil in the process of polymer degradation has been revealed. It is shown that the studied uracil significantly slows down the process of accumulation of hydroperoxides in oxidation of dioctyl phthalate the plasticizer of PVC, which is the cause of a significant slowdown in the rate of decomposition of the plasticized polymer.
\end{abstract}

Keywords: Polyvinyl chloride, thermooxidative dehydrochlorination, antioxidant, 5-hydroxy-6-methyluracil, low toxicity.

\section{INTRODUCTION}

A number of derivatives of uracil is used as drugs in the practical medicine [1]. For some uracils, in particular, for 5-hydroxy-6-methyluracil and 5-amino-6methyluracil, antioxidant activity in reactions of radicalchain oxidation of isopropanol and 1,4-dioxane [2, 3] has been revealed. In this regard, it is of great scientific and practical interest to study the inhibitory efficiency of 5-hydroxy-6-methyluracil in thermooxidative degradation of rigid and plasticized polyvinyl chloride.

\section{EXPERIMENTAL}

The object of the study is uracil derivative 5hydroxy-6-metiluratsil:<smiles>Cc1[nH]c(=O)[nH]c(=O)c1O</smiles>

Thermooxidative dehydrochlorination of rigid and plasticized PVC was performed at $175^{\circ} \mathrm{C}$ in a bubbling type reactor with a stream of oxygen (3.5 liters per hour). Duration of the thermal stability of PVC (T) was determined by the color change of the indicator "Congo red" in the allocation of $\mathrm{HCl}$ during the degradation of the polymer $\left(175^{\circ} \mathrm{C}\right)$ in accordance with GOST 14041 91. The rate of dehydrochlorination of PVC was determined by the same means as in [4]. The kinetics of accumulation of hydroperoxides was estimated by iodometric method [5].

*Address correspondence to this author at the N.M. Emanuel Institute of Biochemical Physics, Russian Academy of Sciences, 4 Kosygin Str., 119334 Moscow, Russia; Tel: 007-8-495-939-71--91; Fax: 007-8-499-137-41-01;

E-mail: Chembio@sky.chph.ras.ru,gezaikov@yahoo.com
Polyvinyl chloride PVC S-7059M was purified by washing with ethanol in a Soxhlet apparatus. Ester plasticizer dioctyl phthalate (DOP) was purified by filtration through a column filled with alumina. 5hydroxy-6-methyluracil ( $99.0 \%$ of main substance) was not subjected to further purification.

\section{RESULTS AND DISCUSSION}

The input of 5-hydroxy-6-methyluracil in rigid PVC in conditions of oxidative degradation leads to a noticeable decrease in the rate of dehydrochlorination of polymer (Figure 1). The maximum decrease in the rate of elimination of $\mathrm{HCl}$ was observed at concentration of uracil equal to $2 \mathrm{mmol}$ per $\mathrm{mol}$ of PVC (critical concentration of antioxidant). Beyond the critical concentration of the antioxidant, an increase in the rate of degradation of the polymer is observed.

The decrease in the rate of thermooxidative dehydrochlorination of polymer in the presence of uracil is observed up to values corresponding to the value of the speed of the thermal elimination of $\mathrm{HCl}$ from $\mathrm{PVC}$ in an inert atmosphere, which is typical for stabilizersantioxidants. The stabilizing efficacy of 5-hydroxy-6methyluracil by the level of reduction rate of thermooxidative dehydrochlorination of PVC is comparable to the industrial efficiency of antioxidant diphenylolpropane (Figure 1).

The problem of stabilization of plasticized PVC is largely associated with the prevention of oxidative decomposition of the plasticizer, since plasticizers, in particular ester plasticizers, in the presence of oxygen become easily engaging in free-radical oxidation reactions, activating the process of elimination of $\mathrm{HCl}$ from the polymer. 


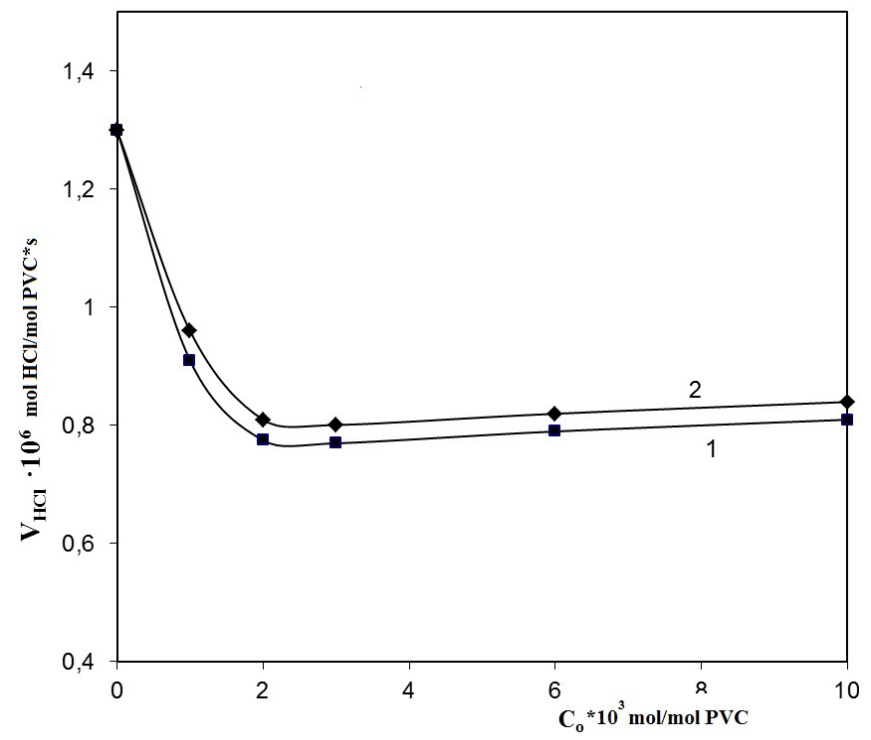

Figure 1: The dependence of the rate of thermooxidative dehydrochlorination of PVC on the content of diphenylolpropane (1) and 5-hydroxy-6-methyluracil (2) $\left(175^{\circ}\right.$ $\mathrm{C}, \mathrm{O}_{2}, 3.5 \mathrm{I} / \mathrm{h}$ ).

The process of thermooxidative dehydrochlorination of PVC plasticized with dioctyl phthalate is accompanied by autocatalysis. The input of 5-hydroxy6-methyluracil in the plasticized polymer leads to a sharp decrease in the rate of thermooxidative dehydrochlorination of polymer and to translation oh the process from autocatalytic to stationary mode (Figure 2).

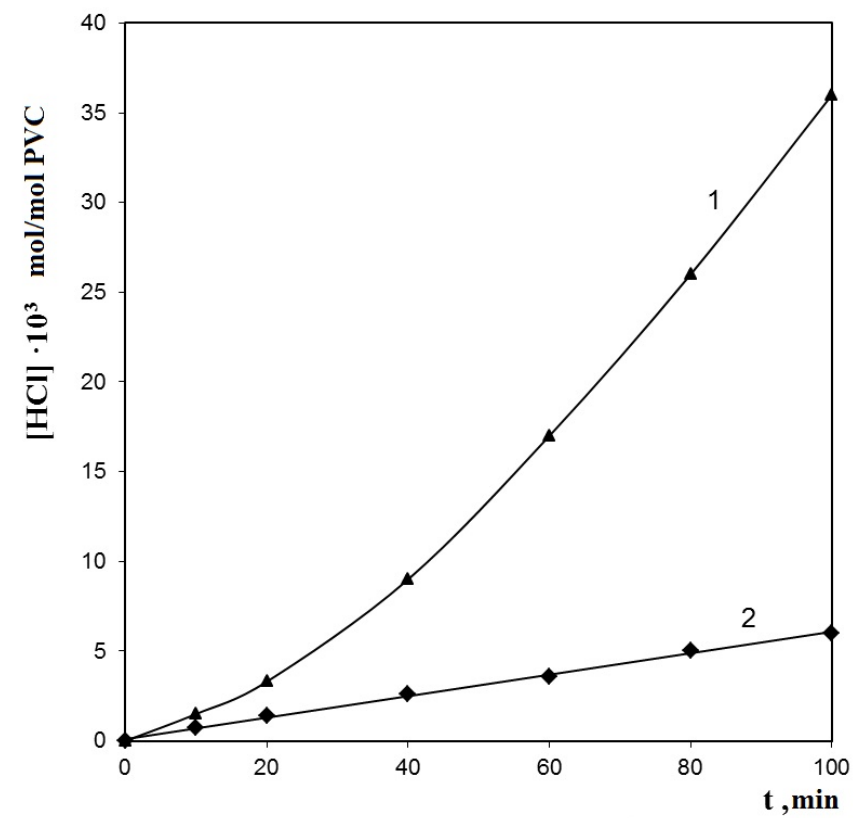

Figure 2: Kinetic curves of the process of dehydrochlorination of PVC plasticized with dioctyl phthalate (40 pbw/100 pbw PVC) in the presence of 5-hydroxy-6methyluracil (content 5-hydroxy-6-methyluracil $1-0 \mathrm{mmol}$ per mol of PVC, 2 - $2 \mathrm{mmol}$ per mol of PVC), $\left(175^{\circ} \mathrm{C}, \mathrm{O}_{2}, 3.5\right.$ $\mathrm{l} / \mathrm{h})$.
The maximum decrease in elimination rate of $\mathrm{HCl}$ from the polymer containing $40 \mathrm{pbw} / 100 \mathrm{pbw}$ of PVC dioctyl phthalate, as in the case of non-plasticized PVC, is observed at concentration of 5-hydroxy-6methyluracil equal to $2 \mathrm{mmol}$ per mol of PVC. Higher concentration of uracil leads to accelerated degradation of the polymer (Figure 3 ).

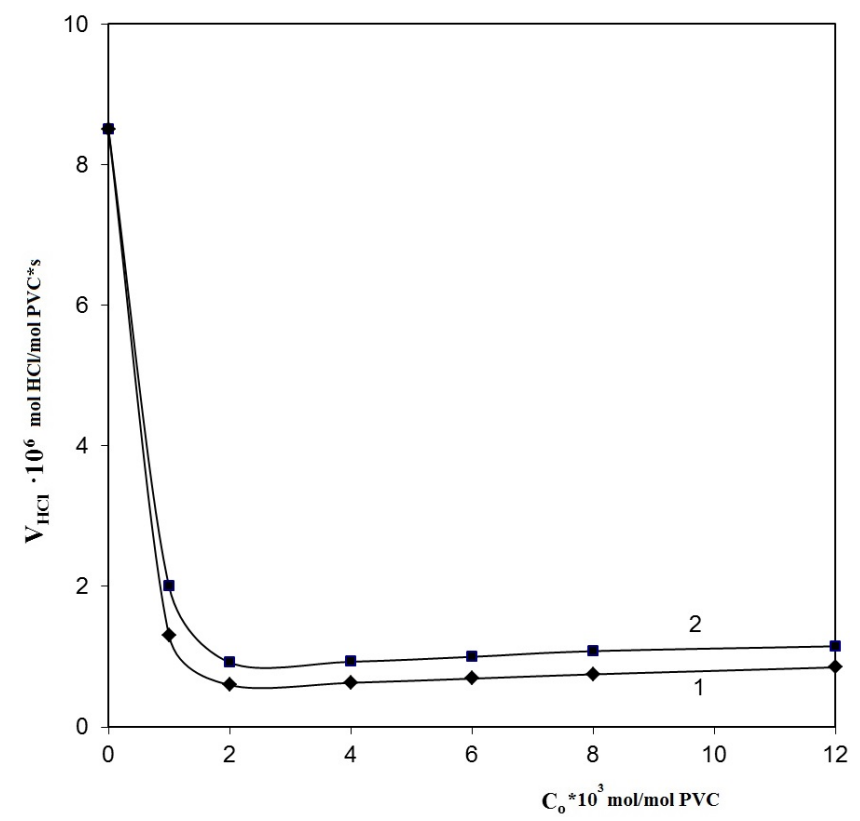

Figure 3: The dependence of the rate of oxidative dehydrochlorination of PVC plasticized by 40 pbw/100 pbw PVC dioctyl phthalate on the content of diphenylolpropane (1) and 5-hydroxy-6-methyluracil $(2)\left(175^{\circ} \mathrm{C}, \mathrm{O}_{2}, 3.5 \mathrm{l} / \mathrm{h}\right)$.

The decrease in the rate of thermooxidative decomposition of plasticized PVC in the presence of 5hydroxy-6-methyluracil is observed up to values corresponding to the rate of the thermooxidative degradation of non-plasticized polymer. Evidently, uracil protects the plasticizer from oxidation, which, in its turn, due to solvation stabilization increases the thermal stability of polyvinyl chloride (a known effect of "echo"-stabilization) [6].

Evidently, as in the case of radical-chain reactions of oxidation of model organic substrate in the presence of uracils [7], an active center in position $\mathrm{N}_{1}$ (link $\mathrm{N}_{1}-\mathrm{H}$ ) in 5-hydroxy-6-methyluracil is responsible for the elementary act of inhibiting oxidation of the ester.

The stabilizing efficacy of uracil is also confirmed by duration of the thermal stability of PVC-compositions comprising metal-containing stabilizer. Additional insertion of 5-hydroxy-6-methyluracil to the plasticized polymer compositions increases the value of "time of thermal stability" by 1.4-1.6 times (Table 1 ). 
Table 1: Time of Thermal Stability of PVC Compounds

\begin{tabular}{|c|c|c|c|c|c|}
\hline \multirow{2}{*}{ Components, Indicator } & \multicolumn{5}{|c|}{ Composition, pbw } \\
\hline & 1 & 2 & 3 & 4 & 5 \\
\hline PVC & 100 & 100 & 100 & 100 & 100 \\
\hline Dioctyl phthalate & 40 & 40 & 40 & 40 & 40 \\
\hline Calcium stearate & 2 & 2 & 2 & 2 & 2 \\
\hline Diphenylolpropane & - & 0,05 & 0,1 & - & - \\
\hline 5-hydroxy-6-methyluracil & - & - & - & 0,05 & 0,1 \\
\hline $\mathrm{T}, \min 175^{\circ} \mathrm{C}$ & 27 & 42 & 49 & 38 & 44 \\
\hline
\end{tabular}

Thermooxidative stability of PVC plasticized by ester is mainly determined by the plasticizer resistance to oxidation. In the process of thermooxidation of ester, the resulting hydroperoxides decompose into radicals that during the degradation of PVC-plasticate have an accelerating influence on the process of dehydrochlorinating of the polymer. In this regard, it is important to evaluate the effect of uracil on the process of accumulation of hydroperoxides during thermooxidation of ester.

The regularities of the process of accumulation of hydroperoxides in thermooxidative decomposition of dioctyl phthalate, the ester plasticizer of polyvinyl chloride, in the presence of 5-hydroxy-6-methyluracil have been revealed. The high efficiency of

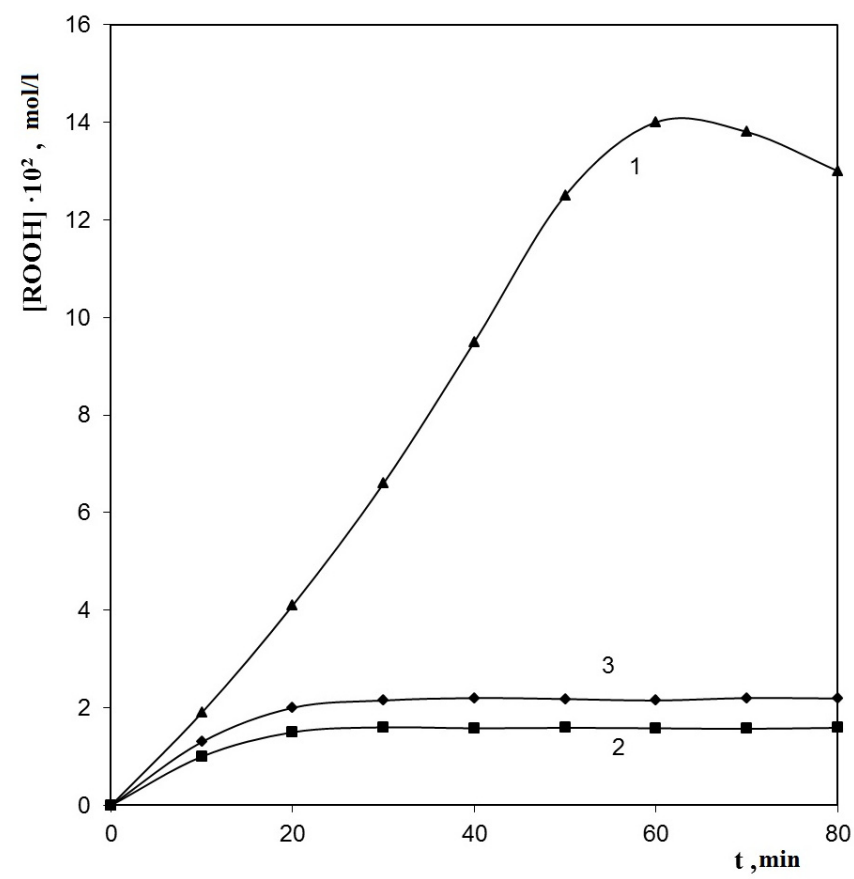

Figure 4: Kinetic curves of accumulation of hydroperoxides in the process of oxidation of dioctyl phthalate in the presence of diphenylolpropane (2), 5-hydroxy-6-methyluracil (3) (concentration of additives is $2 \cdot 10^{-3} \mathrm{~mol}$ per mol of DOF, the original DOF (1), $165^{\circ} \mathrm{C}, \mathrm{O}_{2}, 3.0 \mathrm{l} / \mathrm{h}$ ). antioxidative action of uracil comparable with the efficiency of diphenylolpropane is expressed in a significant reduction of rate of accumulation of hydroperoxides in the oxidation of ester plasticizer (Figure 4).

\section{CONCLUSIONS}

In conditions of thermooxidative dissolution of rigid and plasticized PVC, 5-hydroxy-6-methyluracil is an effective antioxidant-stabilizer and by stabilizing efficiency it is almost equal to industrial phenolic antioxidant diphenylolpropane.

High antioxidant activity of 5-hydroxy-6-methyluracil in the process of destruction of plasticized PVC is explained by the ability of uracil to effectively slow down the process of accumulation of hydroperoxides in the oxidation of ester plasticizer.

High antioxidant activity and low toxicity of 5hydroxy-6-methyluracil (hazard class 4 [8]) allows using it as an antioxidant-stabilizer in formulations of plasticized PVC materials for medical and food purposes.

\section{REFERENCES}

[1] Lazareva DN, Alekhin EK. Immunity stimulants. Moscow: Meditsina, 1985; p. 256

[2] Gerchikov AYa, Garifullina GG, Sultanaeva IV, Krivonogov VP, Mustafin AG, Abdrakhmanov IB. Radical-chain oxidation of isopropyl alcohol inhibited by uracil additives. Pharm Chem J 2000; 34: 543-5. http://dx.doi.org/10.1023/A:1010315415623

[3] Yakupova LR, Sakhautdinova RA, Pankratyev EYu, Safiullin RL. Inhibiting effect of 5-amino-5-methyluracil and its derivatives on the free-radical oxidation of 1,4-dioxane Kinetics and Catalysis 2012; 53: 708-15.

[4] Gabitov IT, Akhmetkhanov RM, Kolesov SV, et al. Bulletin of Bashkir University 2012; 17: 48-51.

[5] Antonovsky VL, Buzlanova MM. Analytical Chemistry of Organic Peroxide Compounds. Moscow: Khimiya, 1978; p. 309. 
[6] Minsker KS, Abdullin MI. The effect of "Echo-stabilization" in thermal PVC. Reports USSR Acad Sci 1982; 263: 140-3.

[7] Dautova IF, Akhatova IV, Safarova IV, et al. Reports of Academy of Sciences 2010; 431(4): 1-3.
[8] Berkhin EB, Lampatov VV, Ul'yanov GP. Effect of 5-hydroxy6-methyluracil and dimetophosphon on tubular secretion of xenobiotics in kidney. Pharmacol Toxicol 1987; 5: 37-8. 